\title{
Direction-selective motion blindness after unilateral posterior brain damage
}

\author{
Olaf Blanke, ${ }^{1}$ Theodor Landis, ${ }^{1}$ Christophe Mermoud, ${ }^{2}$ Laurent Spinelli ${ }^{1}$ and Avinoam B. Safran ${ }^{2}$ \\ ${ }^{1}$ Department of Neurology, University Hospital of Geneva, 24 rue Micheli-du-Crest, 1211 Geneva, Switzerland \\ ${ }^{2}$ Department of Ophthalmology, University Hospital of Geneva, 22 rue Alcide-Jentzer, 1211 Geneva, Switzerland
}

Keywords: brain damage, direction discrimination, human cortex, psychophysics, visual motion

\begin{abstract}
Motion blindness (MB) is defined as the selective disturbance of visual motion perception despite intact perception of other features of the visual scene. $\mathrm{MB}$ is characterized by a pandirectional deficit of motion direction discrimination and is assumed to result from damage to the visual motion pathway, especially area MT/V5. However, the most characteristic feature of primate MT/V5 neurons is not their motion selectivity but their preference for one direction of motion (direction selectivity), which changes incrementally at neighbouring columns. In addition to this microscopic directional organization, studies in nonhuman and human primates suggest that single directions of motion are also coded at a more macroscopic level. We thus hypothesized that if $\mathrm{MB}$ in humans results from damage to direction-selective neurons in the visual motion pathway, posterior brain damage might cause MB which is direction selective, not pandirectional. The present study investigated motion direction discrimination in patients with posterior unilateral brain damage and determined separate psychophysical thresholds for the four cardinal directions. In addition, we analysed whether the direction of erroneous motion perception (i.e. the perception of right motion for upward motion) was random or showed a directional bias. We report three principal findings. First, motion direction discrimination was severely impaired in one or two directions while it was normal in the other directions. This constituted direction-selective MB. Second, MB was characterized not only by a quantitative direction-selective increase in psychophysical thresholds but also by a qualitative impairment of perceiving motion direction systematically in wrong directions. Both findings suggest that the cortical modules specialized for the perception of a single direction of motion might be larger than previously thought. Third, lesion analysis showed that unilateral damage, not only the human homologue of MT/V5 but also to parieto-occipital cortex, leads to MB.
\end{abstract}

\section{Introduction}

Motion blindness (MB or akinetopsia) in humans is characterized by the selective disturbance of visual motion perception despite normal perception of other features within the visual scene such as colour and shape (Poetzl \& Redlich, 1911; Goldstein \& Gelb, 1918; Zihl et al., 1983; Vaina, 1989; see reviews by Grüsser \& Landis, 1991; Zeki, 1991). The associated brain damage is generally extensive and bilateral, including the temporal, parietal and occipital lobe (Zihl et al., 1983, 1991; Vaina et al., 1989). MB is characterized by a pandirectional deficit of motion direction discrimination (MDD), which has also been described in a less severe form subsequent to unilateral brain damage in the central and contralateral visual field (Plant \& Nakayama, 1993; Plant et al., 1993; Barton et al., 1995; Greenlee et al., 1995; Schenk \& Zihl, 1997; Vaina et al., 2001). MB is believed to result from circumscribed damage to direction-selective visual neurons in the cortical visual motion pathway, especially MT/V5 (Nakayama, 1985; Maunsell \& Newsome, 1987; Zeki, 1991). In agreement with this, following the study of Newsome \& Paré (1988) who described a deficit in MDD along the vertical axis, Pasternak and colleagues have described an MDD in the macaque following unilateral (Rudolph \& Pasternak, 1999) or bilateral (Pasternak \& Merigan, 1994) ablation of area MT/V5. However, the latter area contains large amounts of neurons, whose main

Correspondence: Dr Olaf Blanke, Functional Brain Mapping Laboratory, ${ }^{1}$ Department of Neurology, as above.

E-mail: olaf.blanke@hcuge.ch

Received 14 October 2002, revised 27 March 2003, accepted 6 May 2003

characteristic feature is not their motion selectivity (pandirectional) but their preference for one direction of motion (direction selectivity; Dubner \& Zeki, 1971; Zeki, 1974; Baker et al., 1981; Van Essen et al., 1981; Albright et al., 1984). In addition, MT/V5 is organized in a retinotopic fashion (Gattass \& Gross, 1981; Van Essen et al., 1981; Albright \& Desimone, 1987; Maunsell \& Van Essen, 1987). Whereas Albright et al., (1984) showed that direction-selective neurons in MT/ V5 are organized in columns of similar directions and that neighbouring neurons incrementally code different directions, Malonek et al. (1994) have provided evidence that direction in MT/V5 is also coded topographically at a more macroscopic level. It might thus be hypothesized that partial damage to MT+/V5 and adjacent motion areas might lead not only to a motion-selective visual discrimination deficit (pandirectional) as generally stated, but to a direction-selective deficit. Although most previous studies in patients with MB have only described a motion-selective deficit (Plant et al., 1993; Greenlee \& Smith, 1997; Schenk \& Zihl, 1997), Barton et al. (1995) found a predominant deficit in MDD in the ipsilesional direction along the horizontal axis in four of 23 patients. This was also reported by Plant \& Nakayama (1993; case 2) in one patient and confirmed by Vaina et al. (2001) in a group of patients. Because both latter studies did not report thresholds for vertical directions, none of the previous group studies have analysed MDD thresholds for each of the four cardinal directions separately. It is thus not known whether unilateral brain damage leads to direction-selective MB. However, this demonstration seems necessary before arguing that $\mathrm{MB}$ results from predominant damage to directionselective neurons in the visual motion pathway. 
Here we investigated MDD in patients with unilateral brain damage and determined separate psychophysical thresholds for the four cardinal directions. We also analysed whether the direction of erroneous motion perception at the psychophysical threshold (i.e. the perception of right motion for upward motion) was random or showed a directional bias.

\section{Materials and methods}

\section{Patients and control subjects}

Twenty-one acute stroke patients with circumscribed unilateral posterior brain lesions were admitted to the study from a well-defined recruitment area. Only patients suffering from acute vascular strokes were included in the present study in order to avoid as much as possible functional reorganization related to plasticity changes in patients with chronic lesions. Informed consent was obtained from all patients and the study was conducted in conformity with the Declaration of Helsinki. The visual fields of both eyes were tested for all patients with an OCTOPUS 2000R automated perimeter (Interzeag AG, Switzerland). Patients with hemianopia were excluded from the study. Visual acuity was normal or corrected to normal in all patients and healthy subjects. A detailed neuropsychological examination was carried out to test for signs of visual neglect, attention disorders or memory, language or executive disorders. Patients suffering from hemispatial neglect and significant cognitive impairments were excluded from the study. Patients with language disorders but spared comprehension, mild cognitive deficits (attentional or mnestic) or incomplete visual field defects were admitted to the study. Depending on the performance in the motion discrimination task, the patients were classified into two groups: patients who had abnormally elevated psychophysical thresholds for at least one cardinal direction constituted group A; all other patients were in group B (see Results). The patient group A $(n=10)$ consisted of seven males and three females, group B $(n=11)$ of six males and five females. Patient B-7 was lefthanded; all other patients in both patient groups were right-handed. Both groups had approximately similar numbers of patients with right and left hemispheric brain damage (see Tables 1 and 2). Patient A-1 had bilateral brain damage, which was largely predominant in the right hemisphere and was included in the analysis (only right hemispheric damage was used for lesion analysis; Table 1). The 14 control subjects (group C) were approximately matched for age, sex and handedness. The mean age of the patients of group A was $62.1 \pm 13.5$ years, that of group B $59.9 \pm 17.2$ years and that of group C $54.1 \pm 8.5$ years. There was no significant difference between the mean ages of the patient and control groups ( $t$-test for independent samples: A-C, $t_{22}=1.63$,
$\left.P=0.12 ; \mathrm{B}-\mathrm{C}, t_{23}=0.92, P=0.36\right)$, nor was there a difference between the mean age of the two patient groups $\left(t_{19}=0.32, P=\right.$ 0.75). MDD was tested $1.5 \pm 1.2$ months after lesion onset in group A and $0.7 \pm 0.8$ months after lesion onset in group B. There was no significant difference between the mean onsets of testing between the patient groups ( $t$-test for independent samples, $t_{19}=1.66 ; P=0.11$ ). For further demographic and clinical details please refer to Tables 1 and 2 .

\section{Stimuli and apparatus}

Coherent motion stimuli (random dot cinematograms, RDC) were presented on a 20-inch computer monitor (Sony; frame rate $70 \mathrm{~Hz}$, $640 \times 480$ pixels) in black and white in a normally lit room as described previously (Losey et al., 1998; Blanke et al., 2002). Viewing distance was $100 \mathrm{~cm}$. The stimuli were presented in a borderless square of $12 \times 12^{\circ}$ in the central visual field. Each random dot field contained 1000 dots (diameter $0.68^{\circ}$ ). A percentage of the dots was programmed to be displaced with a velocity of $2 \%$ in the tested direction (signal dots) and thus comparable to velocities used in previous studies in groups of brain-damaged patients (Schenk \& Zihl, 1997, 1\%s; Barton et al., 1995, 3.5\%; Vaina et al., 2001, 2.9\%). The percentage of coherence motion $(\% \mathrm{CM})$ was defined as the number of signal dots divided by the total number of dots and multiplied by 100 . The remaining dots were noise dots and plotted at random locations for a random duration giving the impression of flickering dots. Dots moving out of the stimulus area reappeared on the opposite side. The direction of each RDC stimulus in each block was varied randomly between the four cardinal directions (right-left-up-down). An automated staircase algorithm varied the \%CM in the RDC, starting at $100 \% \mathrm{CM}$ (all dots moving in one direction). Subjects were asked to report whether they perceived motion as right, left, up or down. However, patients frequently and repeatedly replied that they did not perceive any directed motion and could not indicate any specific direction. We thus modified the paradigm to the patients' comfort and carried out a five-alternative forced-choice paradigm in which the patients had to reply either the perceived direction or that they did not perceive the direction of motion. This combination of motion direction discrimination and detection was carried out in the brain-damaged patients and the normal subjects in the present study. If the answer of the subject was correct, the \%CM was decreased; if not, $\% \mathrm{CM}$ was increased. Four independent staircases (one for each direction) were randomly interleaved. The four staircases were continued until five response reversals had occurred for each tested direction. The fact that the termination of one directional staircase (i.e. in the case of a pathologically elevated threshold for this direction) would render the remaining directional staircases easier was controlled

TABle 1. Patient demographic and clinical data for group A

\begin{tabular}{|c|c|c|c|c|c|c|c|}
\hline \multirow[b]{2}{*}{ Patient } & \multirow[b]{2}{*}{ Age (years) } & \multirow[b]{2}{*}{ Gender } & \multirow[b]{2}{*}{ Handedness } & \multicolumn{4}{|l|}{ Lesion } \\
\hline & & & & Duration (months) & Side & Location & Visual Field \\
\hline A-1 & 65 & $\mathrm{M}$ & $\mathrm{r}$ & 1.2 & $\mathrm{R}(\mathrm{L})$ & P-O (O) & LIQ (complete) \\
\hline A-2 & 86 & M & $\mathrm{r}$ & 0.7 & $\mathrm{R}$ & $\mathrm{P}-\mathrm{O}$ & Full \\
\hline A-3 & 70 & $\mathrm{~F}$ & $\mathrm{r}$ & 1.2 & $\mathrm{R}$ & $\mathrm{P}-\mathrm{O}$ & LIQ (partial) \\
\hline A-4 & 56 & M & $\mathrm{r}$ & 0.8 & $\mathrm{R}$ & $\mathrm{P}$ & Full \\
\hline A-5 & 59 & M & $\mathrm{r}$ & 2 & $\mathrm{R}$ & $\mathrm{T}-\mathrm{O}$ & LSQ (complete) \\
\hline A-6 & 37 & $\mathrm{~F}$ & $\mathrm{r}$ & 3.2 & $\mathrm{R}$ & P-O-T & LIQ (partial) \\
\hline A-7 & 65 & $\mathrm{~F}$ & $\mathrm{r}$ & 0.4 & $\mathrm{~L}$ & $\mathrm{~T}-\mathrm{O}$ & RIQ (complete) \\
\hline A-8 & 52 & M & $\mathrm{r}$ & 0.5 & $\mathrm{~L}$ & O-P-T & RIQ (partial) \\
\hline A-9 & 56 & $\mathrm{~F}$ & $\mathrm{r}$ & 4.1 & $\mathrm{~L}$ & $\mathrm{~T}-\mathrm{O}$ & Full \\
\hline A-10 & 75 & $\mathrm{M}$ & $\mathrm{r}$ & 0.7 & $\mathrm{~L}$ & $\mathrm{~T}-\mathrm{O}$ & Full \\
\hline
\end{tabular}

M, male; F, female; r, Right-handed; R, right hemisphere lesion; L, left hemisphere lesion; P, parietal; O, occipital; T, temporal; LIQ, left inferior quadrananopsia; RIQ, right inferior quadrananopsia; LSQ, left superior quadrananopsia; RSQ, right superior quadrananopsia. 
TABLE 2. Patient demographic and clinical data for group B

\begin{tabular}{|c|c|c|c|c|c|c|c|}
\hline \multirow[b]{2}{*}{ Patient } & \multirow[b]{2}{*}{ Age (years) } & \multirow[b]{2}{*}{ Gender } & \multirow[b]{2}{*}{ Handedness } & \multicolumn{4}{|l|}{ Lesion } \\
\hline & & & & Duration (months) & Side & Location & Visual Field \\
\hline B-1 & 54 & $\mathrm{~F}$ & $\mathrm{r}$ & 0.3 & $\mathrm{~L}$ & $\mathrm{P}-\mathrm{O}$ & Full \\
\hline B-2 & 56 & $\mathrm{~F}$ & $\mathrm{r}$ & 0.3 & $\mathrm{~L}$ & $\mathrm{P}-\mathrm{O}$ & Full \\
\hline B-3 & 70 & M & $\mathrm{r}$ & 0.5 & $\mathrm{R}$ & $\mathrm{T}-\mathrm{O}$ & LSQ (complete) \\
\hline B-4 & 72 & $\mathrm{~F}$ & $\mathrm{r}$ & 2.9 & $\mathrm{~L}$ & $\mathrm{~T}-\mathrm{O}$ & RIQ (partial) \\
\hline B-5 & 68 & $\mathrm{M}$ & $\mathrm{r}$ & 0.4 & $\mathrm{~L}$ & $\mathrm{~T}-\mathrm{O}$ & RSQ (partial) \\
\hline B-6 & 38 & M & $\mathrm{r}$ & 0.6 & $\mathrm{R}$ & $\mathrm{T}-\mathrm{O}$ & Full \\
\hline B-7 & 25 & $\mathrm{~F}$ & 1 & 0.9 & $\mathrm{R}$ & $\mathrm{T}-\mathrm{O}$ & Full \\
\hline B-8 & 54 & $\mathrm{~F}$ & $\mathrm{r}$ & 0.3 & $\mathrm{R}$ & $\mathrm{O}$ & Full \\
\hline B-9 & 87 & M & $\mathrm{r}$ & 0.5 & $\mathrm{R}$ & $\mathrm{O}$ & LIQ (partial) \\
\hline B-10 & 71 & $\mathrm{M}$ & $\mathrm{r}$ & 0.6 & $\mathrm{R}$ & $\mathrm{P}$ & LIQ (complete) \\
\hline B-11 & 64 & $\mathrm{M}$ & $\mathrm{r}$ & 0.2 & $\mathrm{R}$ & $\mathrm{T}-\mathrm{O}$ & LSQ (complete) \\
\hline
\end{tabular}

M, male; F, female; r, right-handed; 1, left-handed; R, right hemisphere lesion; L, left hemisphere lesion; P, parietal; O, occipital; T, temporal; LIQ, left inferior quadrananopsia; RIQ, right inferior quadrananopsia; LSQ, left superior quadrananopsia; RSQ, right superior quadrananopsia.

as follows. Once one directional staircase was finished, this latter directional staircase was continued (but not used for threshold determination) along with the remaining staircases until five response reversals had also occurred for all remaining directions. Thus, the same number of repetitions was carried out for each tested direction per subject who participated in the study. The mean of the last three reversals was taken as the \%CM threshold. Subjects were instructed to look at the centre of the screen and to refrain from eye movements. Fixation was monitored by the examiner and trials rejected if fixation was not maintained (we can thus not exclude the possibility that small amplitude eye movements might have occurred during some trials). No feedback about correctness of the response was provided. Subjects gave their answer aloud and the examiner recorded the response. The rate of trial presentation was controlled by the examiner and adjusted to patient comfort. Testing took $\approx 20 \mathrm{~min}$ and was carried out in one session.

\section{Threshold analysis}

Psychophysical thresholds were analysed in each tested direction separately in all patients and all healthy subjects. In patients, the horizontal directions were classified and analysed depending on the side of lesion. Thus, the performance of a patient with right hemispheric brain damage for stimuli moving in the right direction was classified as the ipsilesional direction and leftwards moving stimuli as the contralesional direction. In the healthy controls, the performance in the right direction was defined as contralateral and the performance in the left direction as ipsilateral. Based on the results from the healthy subjects, upper $99 \%$ confidence intervals were derived for all tested directions (contralesional, ipsilesional, up, down). In patients, \%CM thresholds falling above these limits were considered pathological threshold elevations. If a patient had a pathologically elevated \% CM threshold in at least one of the tested directions, she or he was considered abnormal and included in group A. The remaining patients constituted group B. Statistical analysis was performed using a two-way ANOVA on the contrast threshold value, which tested the effects of the between-subjects factor experimental group (group A, group B, group C) and the withinsubject factor direction (contralesional, ipsilesional, up, down).

\section{Analysis of directional misperceptions}

Two qualitatively different types of error could occur during MDD: on the one hand, the direction of motion could be erroneously discriminated (i.e. right response for a motion signal which indicates down motion) to produce a directional error (DE). Alternatively, motion direction could be not discriminated at all, resulting in a 'no directed motion' response (NO). The two error types were analysed separately. The total number of wrong responses characterized by the absence of directed motion perception (NO) as well as of DEs were calculated. Direction was classified as for threshold analysis (contralesional, ipsilesional, up, down). For DEs, the sum of all false perceptions of motion direction (into the contralesional, ipsilesional, up, and down directions) were calculated separately. Thus, the number of DEs in the ipsilesional direction was the sum of all motion stimuli moving in the contralesional, up or down directions which were falsely perceived in the ipsilesional direction, etc. Thus, an incorrect ipsilesional response for a contralesional or downward motion stimulus were both defined as an ipsilesional DE. This allowed us to calculate the number of DEs in each tested direction. Statistical analysis was performed using a two-way ANOVA on the number of DEs, which tested the effects of the between-subjects factor experimental group (group A, group B, group C) and the withinsubject factor direction (contralesional, ipsilesional, up, down).

\section{Lesion analysis}

All brain lesions were delineated by magnetic resonance imaging (MRI). MRI was performed with a 1.5-tesla Eclipse system (Marconi Medical Systems, Cleveland, OH, USA). Lesions were drawn on the original MRIs and subsequently normalized into Talairach space (Talairach \& Tournoux, 1988) as described previously (Spinelli et al., 2001). All lesions were mapped onto the left hemisphere, irrespective of the side of brain damage. In patient A-1 who had bilateral but strongly dominant right hemisphere damage, only the latter brain damage was included in the lesion analysis. Three-dimensional rendering and superimposing of the individual lesions was carried out using AVS software (Advanced Visual Systems, MA, USA; see Spinelli et al., 2001).

\section{Results}

\section{Psychophysical thresholds}

All 21 patients completed testing, and results were compared with the results of 14 age-matched healthy subjects. Eleven patients had normal discrimination thresholds (i.e. within 99\% confidence intervals of normals) in all tested directions (group B) and 10 patients had abnormally elevated psychophysical thresholds in at least one tested direction (group A). The number of repetitions per subject carried out to determine the psychophysical thresholds did not differ statistically between the three subject groups (mean $\pm \mathrm{SD}$ : group A, 21.0 \pm 2.9 ; group B, 22.9 \pm 1.9 ; group C, $22.6 \pm 2.6$; $t$-test for independent samples, $\mathrm{A}-\mathrm{B}, t_{9}=-1.395, P=0.196 ; \mathrm{A}-\mathrm{C}, t_{9}=-1.237, P=0.247, \mathrm{~B}-\mathrm{C}$, $t_{10}=0.6531, P=0.528$ ). In group $\mathrm{A}$, a unidirectional deficit in MDD was found in five patients, a bi-directional deficit in one and a 


\section{Psychophysical Thresholds \\ Single Patient Data}
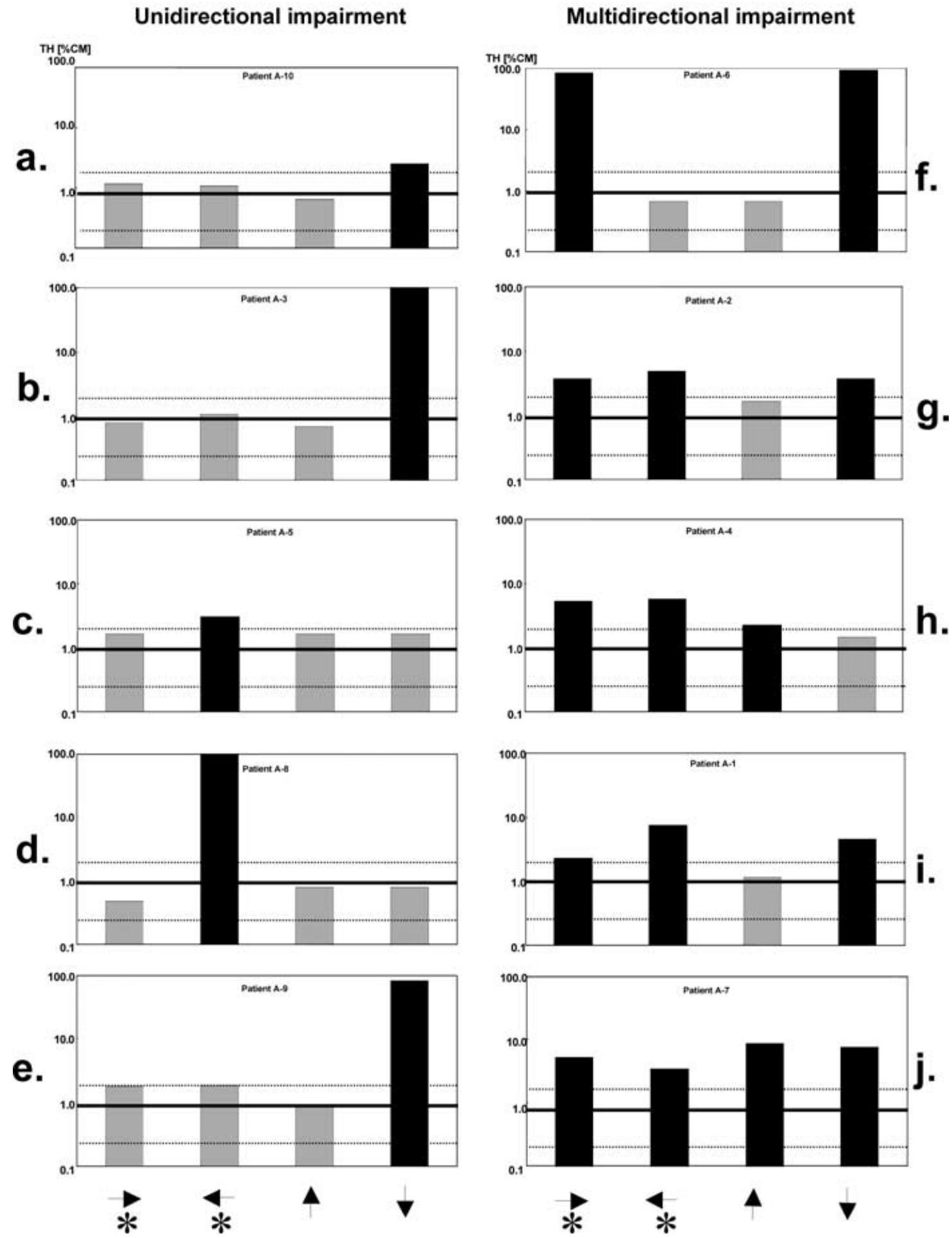

Fig. 1. Psychophysical thresholds for all tested directions are given for all patients from group A. (a-e) The performance of the patients from group A with unidirectional MB. Pathologically elevated psychophysical thresholds are indicated by black columns. Thus, patient A-9 (e) has an abnormally elevated threshold in the down direction (black column), but discriminated all other directions normally. The same was true for patient A-8, but the MDD deficit was found in the ipsilesional direction (d, black column). (f) Patient A-6 from group A with bidirectional MB. Her threshold values were abnormally elevated in the contralesional and down direction, but normal in the other tested directions. ( $\mathrm{g}-\mathrm{j}$ ) The performance of patients with tri- or pandirectional MDD deficits. The performance for each tested direction is indicated by the arrows. The arrow pointing to the right (with asterisks) represents the contralesional direction and the arrow pointing to the left (with asterisks) the ipsilesional direction for all patients. The $y$-axis indicates the psychophysical threshold $(\mathrm{TH})$ in percentage coherent motion (\% CM; logarithmic scale, 0.1-100). Dashed lines depict upper and lower $99 \%$ confidence limits and the thick line the mean as derived from the control group (group C). 
tridirectional in three patients. One patient had a pandirectional deficit. The performance of all patients is illustrated in Fig. 1. Figure 1a-e describes the MDD thresholds of the five patients from group A with a unidirectional MDD deficit. Performance is shown separately for each tested direction. Patient A-9 (Fig. 1e) had an abnormally elevated threshold for down motion $(81.3 \% \mathrm{CM})$, but normal performance for other directions (all \% $\mathrm{CM}<1.8$ ). Similar findings were found for patient A-8 who had an abnormal threshold for motion in the ipsilesional direction $(100 \% \mathrm{CM})$, while MDD was normal in the contralesional, the up and the down directions (all $\% \mathrm{CM}<0.8$; see Fig. 1d). Figure 1f depicts the performance of patient A- 6 with a bidirectional MDD deficit (in the contralesional and down direction) and Fig. $1 \mathrm{~g}-\mathrm{j}$ the performance of patients with tri- or pandirectional MDD deficits. Note that the strength of threshold elevation is especially severe in three patients with a unidirectional MDD deficit (Fig. 1b, d and e) and the patient with a bidirectional MDD deficit (Fig. 1f). In these four patients the one or two pathological thresholds are increased $\approx 100$-fold, whereas the remaining directional thresholds are normal.

Figure 2a depicts separately the means and SEMs of the thresholds of the three subject groups for each tested direction. Note that the psychophysical thresholds for each tested direction were elevated by $\approx 1 \log$ unit for group A. Whereas the effect of the experimental group was significant $\left(F_{2,32}=9.47, P=0.001\right)$, there was no significant effect of direction $\left(F_{3,96}=2.15, P=0.109\right)$, nor a significant interaction $\left(F_{6,96}=1.99, P=0.074\right)$. A post hoc comparison (Scheffé test) revealed that this effect was based on the performance of the patients of group A, who performed significantly more poorly than both normals $(P=0.002)$ and patients from group B $(P=0.003)$. The same post hoc analysis for the main effect of direction did not reveal any significant differences (all $P$-values $>0.10$ ). This indicates that the patients' ability (group A) to discriminate the direction of the moving stimuli was significantly impaired, as compared to group B and C. However, this analysis did not reveal a significant influence of the performance in any of the tested cardinal directions on the global performance.

However, all motion-blind patients had a deficit which predominated in one direction and nearly all of them $(90 \%)$ had normal MDD in at least one direction of motion (Fig. 1). In order to search for a directionselective deficit in the motion-blind patients (group A), statistical analysis was repeated (two-way ANOVA with the factors experimental group and stimulus direction) after reordering the MDD performance of each direction for all patients and healthy subjects. Psychophysical performance was not classified depending on the four cardinal directions (contralesional, ipsilesional, up, down) as for the previous analysis. Instead, the psychophysical performance value obtained for each cardinal direction was ordered, depending on the value of the threshold, from lowest to highest threshold. This is explained graphically in Fig. 3. If the MDD deficit in motion-blind patients is pandirectional and equally pathological for each tested direction, reordering the data in this manner should not lead to a significant effect of direction. Alternatively, if MB is direction-selective or predominates in one or two directions, the statistical effect of motion direction on the global performance could be modified and lead to a significant effect of direction. The results of this procedure are explained in Fig. 3 for two patients from group A (Fig. 3a and b) and for one patient from group B (Fig. 3c). In Fig. 2b the results of this re-ordering procedure are shown for all three subject groups (the threshold which was most strongly elevated is shown on the right and the lowest threshold is depicted on the left; intermediate thresholds are shown in the middle). A two-way ANOVA revealed significant effects of stimulus direction $\left(F_{3,96}=7.90, P<0.001\right)$ as well as experimental group (unchanged as above). Importantly, their interaction was also significant $\left(F_{6,96}=6.58, P<0.001\right)$. A series of post hoc comparisons (Scheffé test) of the direction thresholds indicated an effect only for the

\section{Psychophysical Thresholds Group Data}

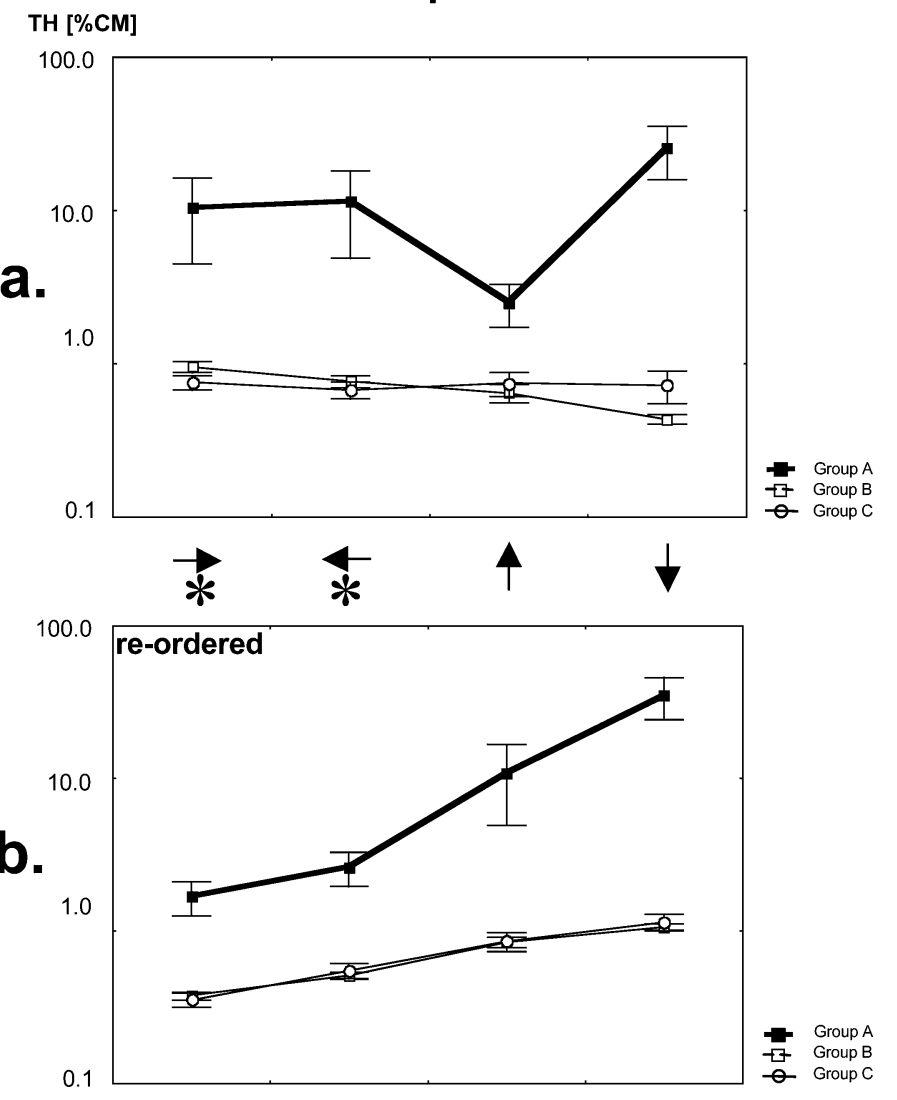

FIG. 2. Psychophysical thresholds for all tested directions are given for the patient and control groups. (a) The mean and SEM of the discrimination threshold (yaxis; logarithmic scale in \%CM) for each tested direction ( $x$-axis) and subject group separately. The direction of the arrow ( $x$-axis) indicates the direction of motion that was to be discriminated. The arrow pointing to the right (with asterisks) represents the contralesional direction and the arrow pointing to the left (with asterisks) the ipsilesional direction. Performance of group A (motion-blind patients) is indicated by the thick line (mean, $\square$ ) and of group B by the thin dashed line (mean, $\square$ ). The performance of the healthy subjects (group C) is depicted by the thin line (mean, $\bigcirc$ ). Note the pathological \%CM values for all tested directions for the patients of group A. Although a significant difference was found for the performance between group A and group B as well as the healthy subjects, no such difference could be detected for the factor direction (see text). (b) The mean and SEM of \%CM is shown again for each subject group separately [each group is coded as in (a)]. However, here the psychophysical performance of each patient is not classified depending on cardinal direction (contralesional, ipsilesional, up, down), but ordered from lowest (left of the figure) to highest (right of the figure) threshold. This classification is therefore independent of cardinal direction (see text, and also Fig. 3 for examples of this re-ordering for individual patients). Regrouping leads, as expected, to higher \% CM thresholds in the right of the figure for all three subject groups. However, statistical analysis shows that the performance in the direction of motion with mostly elevated \% CM thresholds is responsible for the observed differences between motion-blind patients (group A) and healthy subjects (group C) as well as normally perceiving patients (group B).

direction of motion that was most severely impaired (depicted on the right of Fig. 2b; $P$-values from 0.0007 to 0.03 ; all other $P$-values were $>0.68$ ). This indicates that a unidirectional MDD deficit was responsible for the observed differences between motion-blind patients (group A) and healthy subjects (group C) as well as normally perceiving patients (group B).

\section{Directional errors}

Two qualitatively different types of error could occur during MDD: on the one hand, the direction of motion could be erroneously 


\section{grouped depending on cardinal direction}
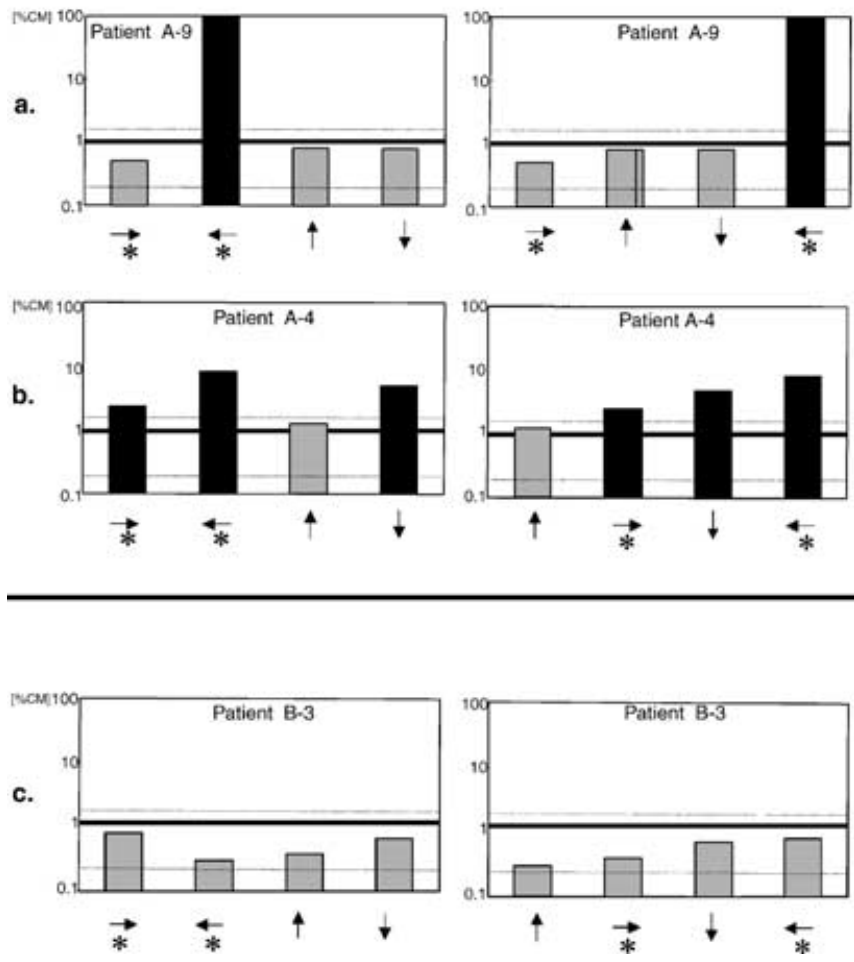

FIG. 3. The psychophysical performance value obtained for each cardinal direction (left column) was ordered, depending on the value of the threshold, from lowest to highest threshold (right column). The results of this procedure are shown for ( $\mathrm{a}$ and $\mathrm{b}$ ) two patients from group A and (c) one patient from group B. See text for further explanation.

discriminated (i.e. right response for a motion signal which indicates down motion) to produce a directional error (DE). Alternatively, motion direction could be not discriminated at all, resulting in a 'no directed motion' response (NO). Although all three groups made the same number of NOs (group A, 15.8 \pm 8.3 ; group B, 17.2 \pm 4.1 ; group $C, 14.1 \pm 4.3$; mean $\pm \mathrm{SD}$; see Table 3 for statistical analysis), patients in group A made significantly more DEs $(8.0 \pm 2.6)$ than either controls $(4.1 \pm 2.4)$ or patients in group B $(2.3 \pm 2.1$; see Table 3$)$. For the two groups of patients, the mean number of DEs is shown in Fig. 4a and compared with the findings in the 14 healthy subjects. As for threshold analysis, a two-way ANOVA on the number of DEs was performed for the effects of the between-subjects factor experimental group (group A, group B, healthy subjects) and the within-subject

TABLE 3. Erroneous motion direction discrimination

\begin{tabular}{lrrr}
\hline Comparison & d.f. & $t$-value & $P$-value \\
\hline NO (A-C) & 22 & 0.637 & 0.53 \\
NO (A-B) & 19 & -0.490 & 0.63 \\
DE (A-C) & 22 & 3.723 & $<0.01$ \\
DE (A-B) & 19 & 5.652 & $<0.001$ \\
\hline
\end{tabular}

Statistical values ( $t$-test for independent samples) for the comparison of the number of directional errors (DEs) and the number of responses that were characterized by the absence of directed motion perception (NO) in both patient groups (groups A and B) and the healthy subjects (group C). Statistical analysis revealed that the motion-blind patients (group A) made the same number of $\mathrm{NO}$ as the patients in group $\mathrm{B}(\mathrm{NO}, \mathrm{A}-\mathrm{B})$ and the healthy controls (NO, A-C). In comparison, the patients of group A made significantly more DEs than patients in group B (DE, A-B) and the healthy subjects (DE, A-C).

\section{Directional Errors Group data}
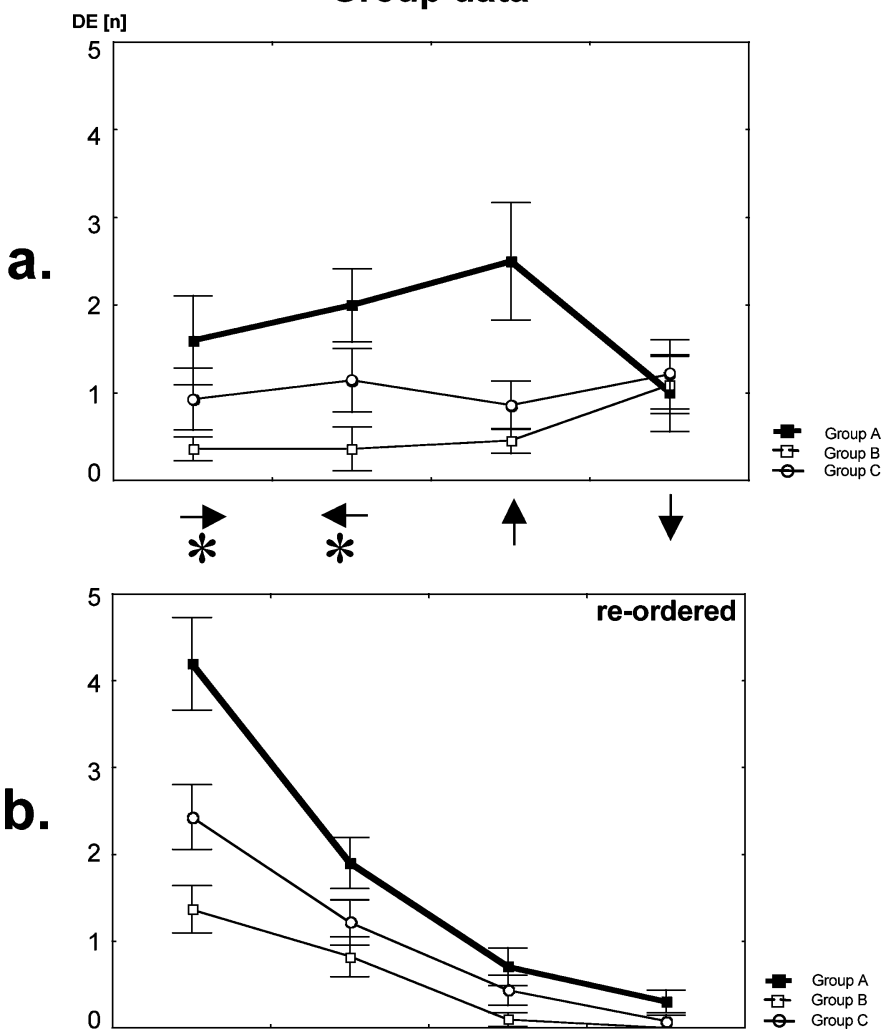

FIG. 4. The total number of DEs in each cardinal direction is shown in (a) for each direction and subject group separately. The direction of the arrows indicates the direction of the DEs. The direction of DEs is indicated at the bottom of the graph (contralesional, ipsilesional, up, down) as in Fig. 2a. Note that the number of DEs in all tested directions (except down) are elevated with respect to group B and the normals (group C). This was found to be significant, although the factor direction was not significant (see text). In (b), the number of DEs of each patient was not classified depending on cardinal direction (contralesional, ipsilesional, up, down), but ordered from highest (left of the figure) to lowest (right of the figure) number of DEs. As for the threshold analysis which is depicted in Fig. 2b, this classification is not dependent on the cardinal direction. The graph depicts the number of DEs separately for each group (A, B and C). Statistical analysis showed that the two directions of motion with the highest number of DEs are responsible for the observed differences between motion-blind patients (group A) and healthy subjects (group C) as well as normally perceiving patients (group B). This demonstrates that motion-blind patients (group A) suffer not only from a reduced capacity to discriminate visual motion but also from an increased likelihood of perceiving a given motion stimulus in a wrong direction.

factor direction (contralesional, ipsilesional, up, down). The effect of the experimental group was highly significant $\left(F_{2,32}=15.83\right.$, $P<0.001)$, but the effect of direction was not $\left(F_{3,96}=2.75, P=\right.$ $0.52)$. There was no significant interaction $\left(F_{6,96}=1.62, P=0.15\right)$. A post hoc comparison (Scheffé test) revealed that this effect was related to the performance of the patients in group A, who performed significantly more poorly than the healthy subjects $(P=0.002)$ and the patients from group B $(P<0.001)$. The same post hoc analysis for the main effect of direction did not reveal any significant differences (all $P$-values $>0.79$ ). This indicates that the motion-blind patients perceived the direction of motion significantly more often in a wrong direction (independent of the four cardinal directions) than did controls and the patients in group B. These data imply that motion-blind patients suffer not only from a reduced capacity to discriminate visual motion (quantitative $\mathrm{MB}$ ), but also from an 


\section{Directional Errors Correspond with Psychophysical Thresholds Single Patient Data}

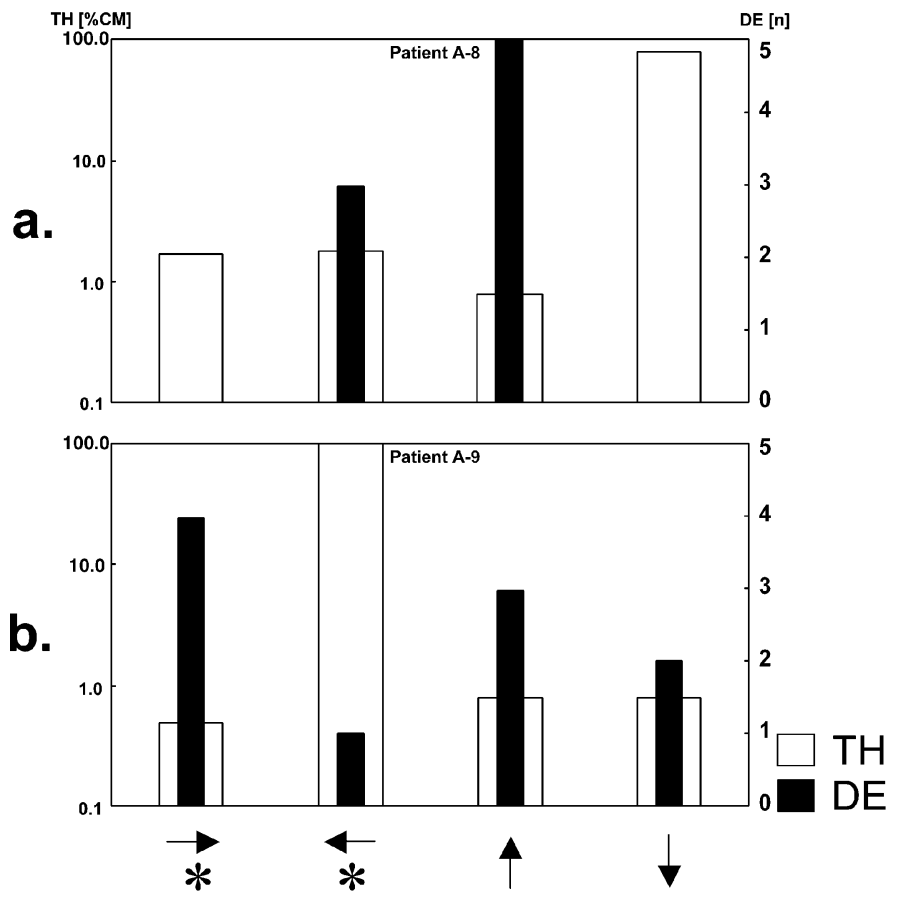

FIG. 5. This figure depicts the number of DEs for each direction separately for two patients with unidirectional motion blindness [(a), patient A-8; (b), patient A-9]. The number of DEs is shown on the right $y$-axis (scale 0-5). Their respective direction is indicated below the graphs. Patient A-8 made a total of eight DEs of which five were in the upwards direction. The remaining three DEs were in the ipsilesional direction. Patient A-9 (b) made 10 DEs of which seven were in two directions (contralesional and up).

increased likelihood of perceiving a given motion stimulus in a wrong direction (qualitative MB).

However, single-data inspection of the DEs in the motion-blind patients showed that DEs often predominated in one or two directions (see Fig. 5). In order to test whether this directional bias of erroneous motion direction perception differed between subject groups, statistical analysis was repeated after reordering the number of DEs. The number of DEs was not classified depending on the four cardinal directions (contralesional, ipsilesional, up, down) as for the previous analysis of DEs. Instead, the number of DEs obtained for each cardinal direction was ordered, depending on the number of DEs, from the direction with the highest to lowest number of DEs (as was done previously for threshold analysis). Again, as for threshold analysis, if DEs in motion-blind patients are pandirectional, reordering the data should not lead to a significant effect of direction. If the direction of DEs predominates in any direction, the statistical effect of motion direction could be modified and lead to a significant effect of direction. The results of this procedure are shown in Fig. $4 \mathrm{~b}$ where the number of DEs is depicted from highest (on the left) to lowest (on the right) for all groups. Statistical analysis was repeated (two-way ANOVA) and main effects of stimulus direction $\left(F_{3,96}=79.3519, P<0.001\right)$ and the experimental group (unchanged as above) were found to be significant, as was their interaction $\left(F_{6,96}=8.3329, P<0.001\right)$. A post hoc comparison (Scheffé test) of the direction of DEs indicated an effect of the two directions into which most DEs were measured (depicted in the two left columns in Fig. $5 \mathrm{~b}$; all $P$-values $<0.001$ ). This indicates that patients in group A not only perceived the direction of motion more often in a wrong direction, but did so systematically in two of four tested directions, than did controls and patients in group B.

Finally, we checked whether there was a systematic relationship between the number of DEs in a given direction and the respective psychophysical thresholds in that direction. This is indicated by the single-patient data as well as the group data. Patient A-8, for example (see Fig. 5a), suffered from unidirectional MB (as defined by psychophysical threshold measurements) and made $100 \%$ (8/8, Fig. 5a) of the total number of DEs in the two directions that had lowest thresholds. Similar findings apply for patient A-9 (Fig. 5b), who made $70 \%$ of the DEs $(7 / 10)$ in the two directions which had lowest thresholds (contralesional, upwards) and only $10 \%$ in the direction of MB (ipsilesional). This relationship between strength of threshold elevations and the number of DEs is also suggested by the data from group A where lowest thresholds and highest number of DEs were in the upward direction (the inverse was true for downward motion perception; compare Figs $2 \mathrm{a}$ and $3 a$ ). In order to test whether a biased choice in favour of the directions of motion with lower thresholds is present for the whole group of motionblind patients, the number of DEs was reordered. Now, the number of DEs in the tested directions was ordered depending on the psychophysical threshold value. The number of DEs in the directions with the two lowest thresholds were grouped and compared with the number of DEs in the directions with the two highest thresholds. This is shown in Fig. 6a for a single patient (compare with the data from the same patient in Fig. 5a), for group A (Fig. 6b), group B (Fig. 6d) and group C (Fig. 6c). As for the reordering depending on the number of DEs in each tested direction (see Fig. 4b), this classification is independent of cardinal direction. Statistical analysis was repeated (two-way ANOVA) and main effects of experimental group (groups A, B and C: $F_{2,32}=21.439, P<0.001$ ) and direction (the two directions with low against the two directions with high psychophysical thresholds: $F_{1,32}=6.6029, P=0.015$ ) were found to be significant. The interaction between the two factors showed a trend $\left(F_{2,32}=2.5147, P=0.09\right)$. A post hoc comparison (Scheffé test) of the direction indicated a significant effect $(P=0.01)$ and the post hoc comparison for the group factor revealed significant differences only for the motion-blind patients (group A, Fig. 6b; all $P$-values $<0.001$ ). This indicates that patients with MB not only perceived the direction of motion more often in wrong directions, but misperceive the direction of motion in the directions with lowest thresholds (Fig. 6b).

\section{Lesion analysis}

Lesion location and extent were determined with MRI in all patients. All lesions were mapped onto the left hemisphere. The comparison of the superimposed lesion plots of the 10 motion-blind patients (group A, top of Fig. 7a) with the 11 patients from group B (bottom of Fig. 7a) showed that different posterior brain areas were damaged in the two groups (see Fig. 7a). Lesion volume did not differ significantly between the two patient groups (group A, $14.0 \pm 3.7 \mathrm{~cm}^{3}$; group B, $16.4 \pm$ $4.3 \mathrm{~cm}^{3}$; mean $\pm 1 \mathrm{SEM}$; $t$-test for independent samples, $t_{19}=-0.415$, $P=0.683$ ). Whereas lesion overlap in the patients of group B centred on one area (green arrow in Fig. 7a), our analysis revealed two discrete areas in the motion-blind patients (red and yellow arrows, Fig. 7a).

One overlap region across patients of group A was situated at the occipitotemporal junction and is indicated in Fig. 7a by the red arrow. The location of this area is concordant with previous anatomical lesion studies in patients with MB (Barton et al., 1995; Greenlee et al., 1995; Schenk \& Zihl, 1997; Vaina et al., 2001). Lesion location and extent was also determined in stereotaxic coordinates (Talairach \& Tournoux, 1988). The stereotaxic mean and range of this overlap region are given in Table 4. Stereotaxic analysis showed that this overlap area is in close proximity to the stereotaxic activation site of area MT+/V5, as proposed by numerous neuroimaging studies (Watson et al., 1993; 


\section{Directional Errors Correlate with \\ Psychophysical Thresholds \\ Group Data}
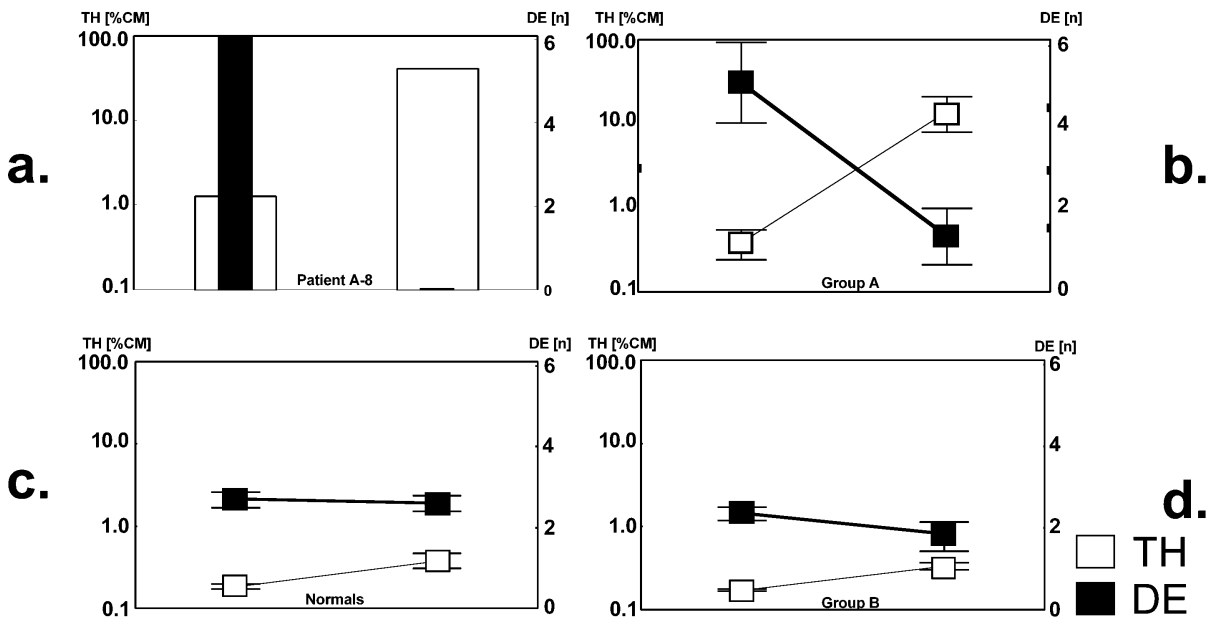

FIG. 6. The direction of motion blindness corresponds with the direction of erroneous motion perception. (a) The same patient is depicted as in Fig. 5a. The number of DEs is grouped depending on the direction-discrimination threshold. In comparison with Fig. 5a, the number of DEs in the directions with the two lowest thresholds were grouped and compared with the number of DEs in the directions with the two highest thresholds. This was done for all patients and healthy controls in order to test whether a biased choice in favour of the better (and normally) perceived directions of motion is present for the motion-blind patients. Note that the inverse correspondence between direction of threshold elevation as suggested in the single motion-blind patient (A-8) is also found in group A (b). This was not the case in groups B (d) and C (c). Symbols: black, number of directional errors; white, psychophysical threshold in \%CM.

de Jong et al., 1994; Dupont et al., 1994, 1997; Tootell et al., 1995; Goebel et al., 1998). The second overlap region in group A was located in the posterior parietal cortex and is indicated by the yellow arrow (Fig. 7a). It was situated close to the parieto-occipital sulcus including the precuneus and the cuneus (see Fig. 6a; stereotaxic mean and range are given in Table 4). This region is located close to an area that has been shown to be involved in normal visual motion perception in the frontal plane as well as visual motion in depth (de Jong et al., 1994; Goebel et al., 1998; Sunaert et al., 1999). Given the strong and direction-selective MDD deficits in patients A-3, A-6, A-8 and A-9 (uni- or bidirectional MDD deficit; compare Fig. 1b, d, e and f), the lesions as defined by MRI are shown for each of these latter patients separately in Fig. 8 (in patient A-9, Fig. 8a, the reconstructed MRI as used for group lesion analysis is shown, because the original MRI was no longer available). In one patient the lesion included the first overlap region (MT+/V5; Fig. 8a) and in two patients brain damage included the second overlap region (Fig. $8 \mathrm{~b}$ and c). In one patient brain damage was mainly observed in the occipital cortex, with parietal and temporal extensions (Fig. 8d).

Lesion overlap in group B centred in a different region in the ventral occipito-temporal cortex that included the anterior cuneus and the lingual gyrus (green arrow, Fig. 7a). The stereotaxic coordinates for this overlap region are given in Table 4 . A second very small region of overlap was found in group B in the inferior occipital gyrus [small red area in Fig. 7a; see horizontal stereotaxic position of -10 (z-axis)]. Figure $7 \mathrm{~b}$ depicts the two overlap regions in group A (depicted in blue) in three dimensions and distinguishes them spatially from the overlap region in group B (green).

\section{Discussion}

\section{Direction-selective threshold elevations}

Motion blindness is believed to result from circumscribed damage to direction-selective visual neurons in the cortical visual motion pathway, especially MT/V5 (Zihl et al., 1983; Nakayama, 1985;
Maunsell \& Newsome, 1987; Zeki, 1991). In monkey, the importance of this pathway and MT/V5 in the perception of motion direction has been demonstrated by single-unit recordings (Dubner \& Zeki, 1971; Zeki, 1974; Baker et al., 1981; Van Essen et al., 1981; Albright et al., 1984) showing that MT/V5 contains large numbers of directionselective neurons. These neurons are densely organized in columns of cells tuned to similar directions, whose directional preference changes incrementally at neighbouring columns. Microstimulation of MT/V5 neurons (Salzman et al., 1990, 1992) and medial superior temporal (MST) neurons (Celebrini \& Newsome, 1995) has extended these findings. In an MDD task, microstimulation in both areas systematically modified a monkey's choice that depended on the neuron's directional preference. Further studies have shown that, in addition to this columnar directional organization, area MT/V5 is organized macroscopically in a retinotopic fashion (Gattass \& Gross, 1981; Van Essen et al., 1981; Albright \& Desimone, 1987; Maunsell \& Van Essen, 1987) suggesting that, in combination with the columnar directional organization, all directions of motion are represented at each retinal location.

In accordance with this functional organization, many studies in humans have presented evidence in favour of retinotopically organized motion processing. Thus, human lesion studies (Plant et al., 1993; Barton et al., 1995; Greenlee \& Smith, 1997; Schenk \& Zihl, 1997) and studies using cortical electrical stimulation (Blanke et al., 2002) or transcranial magnetic stimulation of extrastriate cortex (Beckers \& Hömberg, 1992; Hotson et al., 1994; Beckers \& Zeki, 1995) were able to measure MDD deficits which were confined to the visual field contralateral to brain damage or contralateral to (electrical cortical or transcranial magnetic) stimulation. More recently, the retinotopic organization of human MT+/V5 has also been demonstrated by fMRI studies (e.g. Tootell et al., 1995; Dukelow et al., 2001; Huk et al., 2002).

The main focus of the present investigation was to find out whether circumscribed unilateral brain damage to human extrastriate cortex might lead to a functional deficit in the central visual field reflecting the 


\section{Lesion Analysis}
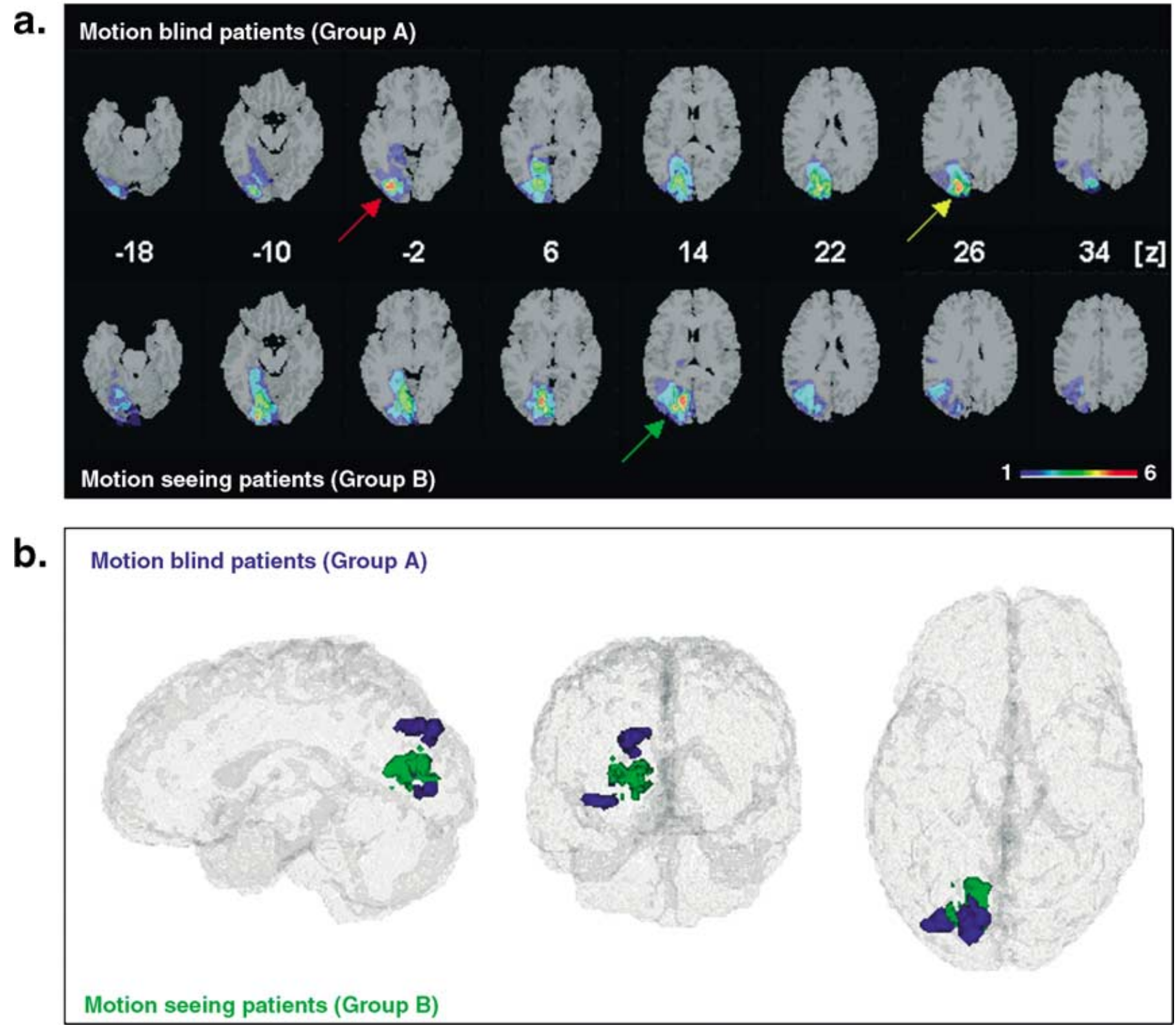

FIG. 7 Lesion analysis of patients with and without motion blindness. (a) Overlap plots for both patient groups. The number of overlapping lesions is indicated by colour, from blue $(n=1)$ to red $(n=6)$. The centre of overlap is indicated in red for patients in group A (top) and B (bottom). The Talairach coordinates of the transverse sections are given in the middle of the figure ( $z$ coordinates). The brain damage in the motion-blind patients (group A) is depicted in the upper part and that of group B in the lower part of (a). Note that two centres of overlap were found in motion-blind patients. One area was localized at the temporo-occipital junction in close proximity to MT+/V5 (red arrow; see Table 2 for stereotaxic coordinates), the other in the posterior parietal cortex (yellow arrow). Both overlap areas were anatomically distinct from the centre of overlap in patients from group B, which is shown at the bottom of (a). This latter area was localized on the cuneus and lingual gyrus and is indicated by a green arrow. (b) Three-dimensional reconstruction of the lesion overlap zones. Lateral (left), posterior (middle) and top view (right) of the centres of overlap in motion-blind patients (blue) and motion-seeing patients (green). Lesion overlap is shown in a transparent brain volume.

main characteristic of area MT+/V5: direction selectivity. Although some previous evidence suggests that this might be the case (Shipp et al., 1994; Barton et al., 1995), this had not been systematically investigated in groups of patients with posterior brain damage. Greenlee \& Smith (1997) measured MDD along the four cardinal directions, but only reported global values. Other studies tested MDD only along the horizontal axis (Plant et al., 1993; Schenk \& Zihl, 1997). However, Plant \& Nakayama (1993) and Barton et al. (1995) described pathological MDD in the ipsilesional direction while MDD in the contralesional direction was normal (i.e. horizontal directional anisotropy). This finding was confirmed by Vaina et al. (2001). (The psychophysical thresholds values for vertical directions were not analysed quantitatively in the three latter studies.) However, for the patients with horizontal directional anisotropy, Barton et al., 1995, page 669) noted that 'thresholds for vertical motion discrimination were also elevated'. This suggests the presence of tridirectional MB in 
TABLE 4. Lesion overlaps

Stereotaxic coordinates $(\mathrm{mm})$

\begin{tabular}{llll}
\cline { 2 - 4 } Group & $X$ & $Y$ & $Y$ \\
\hline A, lateral occipito-temporal & $29(20-38)$ & $79(72-86)$ & $1(-4$ to +2$)$ \\
A, posterior parietal & $13(3-23)$ & $83(71-95)$ & $26(20-32)$ \\
B, ventral occipito-temporal & $14(0-28)$ & $76(59-93)$ & $9(4-14)$ \\
\hline
\end{tabular}

Stereotaxic location of the two overlap regions in group A and the overlap region in group B. Mean as well as overlap surface (range) are given for the maximal overlap areas.

Barton and colleagues' patients and is comparable to MDD deficits in some of the present motion-blind patients. However, by analysing the discrimination thresholds for each cardinal direction separately, our data show that MB is not pandirectional or tridirectional, but that $\mathrm{MB}$ significantly predominates in one of the four cardinal directions for all patients of group A; hence the term 'direction-selective MB'. Direction selectivity was especially evident in some of the present patients with uni- or bi-directional $\mathrm{MB}$ whose $\mathrm{MB}$ was characterized by an $\approx 100$-fold increase in thresholds in one or two directions, but normal thresholds in the remaining directions. Although psychophysical studies in healthy subjects have suggested previously that the human visual cortex processes direction-selective signals (e.g. Levinson \& Sekuler, 1976; Mather, 1980), neural correlates have only recently been detected. These latter results were based on fMRI measurements of motion opponency (Heeger et al., 1999) and direction-selective adaptation (Huk et al., 2001) and found in MT+/V5 and a number of other extrastriate areas. The present data corroborate and extend these previous neuropsychological, psychophysical and neuroimaging findings. The demonstration of direction-selective and unidirectional MB suggests that this clinical condition might result from selective or predominant damage to neurons in the cortical motion pathway tuned to the direction of motion that the subject no longer discriminates (Dubner \& Zeki, 1971; Zeki, 1974; Baker et al., 1981; Van Essen et al., 1981). Accordingly, we conjecture that neurons encoding intact directions of motion are less impaired or unimpaired, leading to normal MDD performance for these directions. However, selective damage to cells tuned to one direction of motion seems rather improbable because neurons in, e.g., MT/V5 are densely organized in directional columns, whose directional preference changes incrementally at neighbouring columns (Albright et al., 1984). However, Malonek et al. (1994) have shown that motion direction in MT/V5 is also coded at a more macroscopic level in neuronal clusters of up to $1 \mathrm{~mm}^{2}$. Evidence from intracranial stimulation of human extrastriate cortex further suggests that modules coding single directions of motion might even be as large as $\approx 1 \mathrm{~cm}^{2}$ (Blanke et al., 2002). These authors have induced unidirectional transient MB by focal electrical stimulation of MT+/V5 and of other extrastriate sites extending beyond MT+/V5: MDD in one direction was completely abolished by electrical stimulation at a given extrastriate site, while the perception of other motion directions at the same site remained intact. The present results following chronic focal brain damage would argue for even larger neuronal modules that mediate the perception of single directions of motion. Although Newsome and colleagues (Newsome et al., 1995; Shadlen et al., 1996; Parker \& Newsome, 1998) have shown that the activity of single direction-selective MT/V5 neurons is closely related to MDD in monkeys they suggested, based on simulations, that directional modules 'are probably composed of at least 100 neurons, but may include many times this number' (Shadlen et al., 1996; page 1499). The present data on psychophysical threshold measurements in human brain-damaged observers are in agreement with this hypothesis, but suggest that the size of the direction-selective modules (or neuronal populations) might be much larger than previously thought. It is thus conceivable that direction-selective MB (especially in the four patients with severely elevated thresholds) might have resulted from predominant damage to neuronal populations in MT+/V5 (Malonek et al., 1994) that code for the direction which the subject cannot discriminate anymore. Although stereotaxic lesion analysis revealed damage to MT+/V5 in the motion-blind patients, the known directional and retinotopic organization of MT/V5 and the fact that naturally occurring brain damage happens by chance, has low areal selectivity and is generally more extensive than experimental lesions in animals (Damasio et al., 2000), make it rather unlikely that direction-selective MB results from selective interference with MT+/V5. Brain damage almost certainly extented over several neighbouring visual motion areas and might have damaged direction-selective modules upstream and/or downstream from MT+/V5. Based on the directional selectivity of the MDD deficit in the present patients, it might thus be hypothesized that the visual motion pathway may consist of motion areas in which cells are macroscopically distributed according to preferred direction. To our knowledge such an organization, apart from the results by Malonek et al. (1994), has not yet been described in primate visual cortex. Alternatively, direction-selective MDD deficits might be caused by relative predominant coding for one direction of motion in each MT+/V5, which has been described in macaque MT/V5 along the horizontal axis (Dubner \& Zeki, 1971). The unilateral loss of one MT+/V5 in the present patients might then lead to a directionselective MDD deficit caused by predominant directional coding of the contralesional, undamaged MT+/V5. This latter mechanism might account for patients suffering from mildly elevated MDD thresholds, such as in patients A-5 and A-10. However, the direction-selective $\approx 100$-fold increases in MDD thresholds in some of the present patients with normal MDD in the remaining directions suggests that the latter mechanism is rather unlikely to account for these patients' performance. Nevertheless, given the presentation of the motion stimulus in the central visual field, a significant contribution of the contralesional, undamaged MT+/V5 to the patients' MDD deficit cannot be excluded.

\section{Erroneous motion perception}

Although MT/V5 neurons are direction selective, they are broadly tuned and respond sometimes to directions of motion that are $<90^{\circ}$ apart (Dubner \& Zeki, 1971; Zeki, 1974; Albright et al., 1984; Maunsell \& Van Essen, 1987). Based on this broad tuning, a unidirectional stimulus excites a widely distributed population of neurons. Accordingly, it has been assumed that MDD in monkey is guided by information contained in direction-selective modules, which include cells whose optimal direction of motion is different from the one being discriminated (Newsome et al., 1995; Shadlen et al., 1996; Parker \& Newsome, 1998; Britten et al., 1992). Although the signalling properties of individual neurons in such direction-selective modules (or neuronal populations) overlap substantially, this redundancy ensures performance against sources of noise and neuronal loss as well as increases its computational speed (Shadlen et al., 1996).

FIG. 8 Magnetic resonance imaging (MRI) of the four patients with severe direction-selective motion blindness. (a) The reconstructed 3-D MRI of patient A-9 with a focal lesion of the left occipito-temporal cortex. (b) T2-weighted MRI of patient A-3, depicting brain damage to the right medial parieto-occipital cortex. Brain damage was more extensive in patient A-6 (c; T2-weighted MRI) and included the right medial and lateral parieto-occipital cortex as well as right frontal cortex. (d) Brain damage in patient A-8 was confined to the medial occipital cortex with extensions into temporal and parietal cortex. 

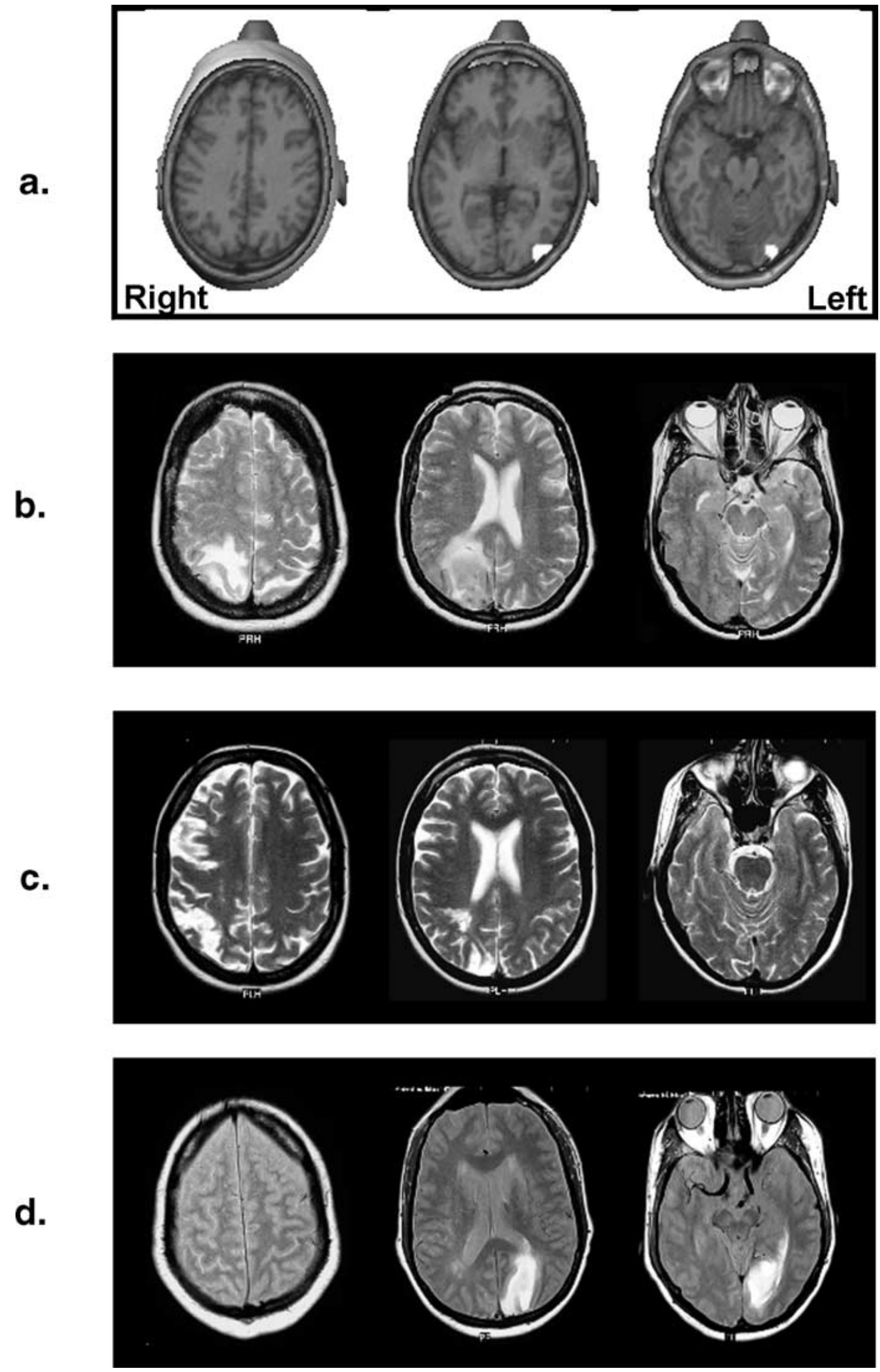
Applied to healthy human subjects as well as patients with unidirectional $\mathrm{MB}$, this model predicts that a motion stimulus in a given direction will concomitantly activate neurons whose optimal direction of motion is different from the one being discriminated. In the present study, the combination of direction-selective psychophysical threshold elevations with direction-selective directional errors in group A suggests that the activation of neurons which encode the direction of motion for which the patient is blind might be outweighed by the concomitant activation of neurons which preferentially code other directions. The higher number of DEs in group A patients provides support for this hypothesis. Most importantly, DEs in group A were shown to be direction-selective. In conclusion, these data suggest that $\mathrm{MB}$ is not only characterized by a quantitative direction-specific increase in psychophysical threshold measurements, but also leads to a qualitative impairment characterized by direction-specific misperceptions during MDD. Direction-selective DEs have also been described in a severely motion-blind patient with bilateral posterior brain damage (Shipp et al., 1994; patient initially described by Zihl et al., 1983). In addition, small directional biases during MDD have also been described in healthy subjects (Raymond, 1994; Gross et al., 1998) and visual inspection shows that it was also found for subjects in group $\mathrm{C}$ and patients in group B in the present study (see Fig. 4). It could thus be argued that DEs in motion-blind patients (group A) merely reflect this latter directional bias. However, two arguments speak against this interpretation. Firstly, statistical analysis showed that only the motion-blind patients systematically misperceived motion direction in two of four tested directions, whereas DEs in healthy controls (group C) and brain lesioned controls (group B) were found to be randomly distributed over all tested directions. Secondly, only in the motion-blind patients did the statistical analysis suggest an inverse relationship between the number of DEs in a given direction and its respective threshold value. Although statistical analysis only showed a trend for the interaction between both latter variables, our data suggest that the two variables relate functionally to each other. It could also be argued that this functional relationship might be caused by a methodological artefact (a patient who always responds 'right' independently of which motion direction is shown would have a normal threshold for rightward motion but pathological thresholds for the other directions). However, during testing we never encountered a patient who responded in this fashion. Such patients are likely to suffer from other cognitive deficits that were searched for in an extensive neuropsychological examination that was carried out prior to motion testing (see Materials and methods). A voluntary bias (especially for testing near the psychophysical threshold) is also unlikely because the different directions were tested in random fashion in parallel order. In addition, whereas most of the motion-blind patients realized their difficulties in MDD they were never aware of a directional motion deficit during testing or during situations in everyday life (as noted for the patient reported by Zihl et al. (1983, 1991) who suffered from severe MB related to bilateral brain damage). Thus, direction selectivity and inverse relationship of DEs with psychophysical thresholds are likely to be functional deficits in the motion-blind patients. Finally, direction-selective DEs have also been described in a single patient with left medial occipital and right parieto-occipital brain damage, but residual visual capacities in his blind hemifield (blindsight; patient GY, Blythe et al., 1987; Zeki \& ffytche, 1998). However, inspection of the reported deficit of patient GY shows that, despite this behavioural similarity, there are many important phenomenological and behavioural differences as well as methodological and clinical differences between former studies and the present investigation. Most importantly, we show direction-selective DEs and threshold increases in a group of 10 patients and dissociate this behavioural pattern from a control group of patients with medial occipital damage similar to patient GY (group B) who shows neither pathological threshold elevations nor pathological increase in DEs. In addition, investigations in patient GY were carried out many years following brain damage ( $>11$ years) allowing for extensive reorganization, whereas the examination of the present patients was carried out shortly after the occurrence of brain damage. Finally, patient GY also suffered from additional right parieto-occipital brain damage (partially concordant with the parieto-occipital overlap region in group A; see Brent et al., 1994). It can thus not be excluded that this right hemispheric brain damage influenced his MDD performance. In conclusion, these data suggest that quantitative and qualitative direction-selective MB is linked to extrastriate cortical damage. Most models of MDD hypothesize that the perception of motion direction results from the relative difference between modules tuned to different directions (e.g. Mather, 1980; Simoncelli \& Heeger, 1998). In accordance, we speculate that direction-selective cell loss in MT+/V5 and/or other visual motion areas in motion-blind patients leads to a pathological imbalance between the different directional populations disfavouring the population that codes for the direction of MB: populations encoding for less impaired directions of motion are favoured and their stronger relative activation might lead to the illusory and erroneous perception of a direction of motion that was not shown.

\section{Damage to the lateral occipito-temporal cortex or to the posterior parietal cortex leads to motion blindness}

The present lesion analysis shows that brain damage in motion-blind patients is anatomically distinct from brain damage in motion-seeing patients. Unexpectedly, brain damage in patients with MB centred in two discrete areas: in the lateral temporo-occipital cortex and in the parieto-occipital cortex. This suggests that brain damage to either region leads to direction-selective $\mathrm{MB}$ (as defined in the present study) and suggests that both regions are involved in the discrimination of motion direction.

Our data confirm previous anatomical studies in patients with MB which have suggested that damage to the lateral temporo-occipital cortex causes MB (Barton et al., 1995; Greenlee et al., 1995; Schenk \& Zihl, 1997; Vaina et al., 2001). However, stereotaxic comparison of the site of brain damage in normalized Talairach space (Talairach \& Tournoux, 1988) with the location of visual motion areas as defined by fMRI and PET (in healthy subjects) has not been reported previously. The present study shows that lateral temporo-occipital brain damage in motion-blind patients is situated in close proximity to area MT+/V5 as defined anatomically (Dumoulin et al., 2000) and functionally by neuroimaging studies (Watson et al., 1993; de Jong et al., 1994; Dupont et al., 1994; Tootell et al., 1995; Goebel et al., 1998) as well as intracranial electrical stimulation (Blanke et al., 2002).

However, our analysis shows that damage to not only MT+/V5, but also to the posterior parietal cortex, may lead to MB. Previous lesion studies in patients with MB have not implicated this posterior parietal region in visual motion perception (Barton et al., 1995; Greenlee et al., 1995; Schenk \& Zihl, 1997) and Vaina et al. (2001), who investigated the effects of different sites of brain damage on MDD, did not investigate patients with damage to the posterior parietal cortex. However, several neuroimaging studies have shown that this region is strongly activated by visual motion in the frontal plane as well as in depth (de Jong et al., 1994; Goebel et al., 1998; Sunaert et al., 1999). Additional neuroimaging evidence for a potential role of this posterior parietal area in mediating motion perception was provided by a PET study (Shipp et al., 1994). This study examined brain activation in response to consciously perceived and correctly discriminated motion direction in the severely motion-blind patient who was initially 
reported by Zihl et al. (1983). In this patient with bilateral damage to MT+/V5 (Zihl et al., 1991), the posterior parietal cortex, concordant with the second lesion overlap region in the present study, was found to be the region most activated by motion stimuli which were discriminated correctly in $\approx 80 \%$ of the trials (Shipp et al., 1994). In line with this evidence, our results point to a significant contribution of the parieto-occipital cortex in motion perception. Its stereotaxic location and anatomical location at the parieto-occipital sulcus suggests that this region might represent or include the human homologue of macaque areas V6 and V6A (Shipp et al., 1996; Galetti et al., 1999a,b), which have been shown to be involved in motion perception and to harbour direction-selective neurons (Galetti et al., 1999a,b). Lesion location in the four patients with severe uni- or bi-directional MB further suggests that damage, suggested by group analysis, to either area might lead to direction-selective MB. Although the functional and anatomical differences between the lateral temporo-occipital cortex and parieto-occipital cortex make it very likely that the functional consequences following cortical damage to either region should differ, the present findings show that unilateral damage to either part of cortex also has similar functional consequences as measured in the current study.

\section{Acknowledgements}

This work was supported by a grant from the Swiss National Science Foundation (SNF grant 3100-065232.01 and 3100-067874.02). We thank C. de Weisse and D. Desangles for technical help and C. Mohr and M. M. Murray for comments on a previous version of the manuscript.

\section{Abbreviations}

$\% \mathrm{CM}$, percentage of coherence motion; $\mathrm{DE}$, directional error; $\mathrm{MB}$, motion blindness or akinetopsia; MDD, motion direction discrimination; MRI, magnetic resonance imaging; MT, middle temporal area; NO, 'no directed motion'; $\mathrm{RDC}$, random dot cinematogram.

\section{References}

Albright, T.D. (1984) Direction and orientation selectivity of neurons in visual area MT of the macaque. J. Neurophysiol., 52, 1106-1130.

Albright, T.D. \& Desimone, R. (1987) Local precision of visuotpic organization in the middle temporal area (MT) of the macaque. Exp. Brain Res., 65, 582592.

Albright, T.D., Desimone, R. \& Gross, C.G. (1984) Columnar organization of directionally selective cells in visual area MT of the macaque. J. Neurophysiol., 51, 16-31.

Baker, J.F., Petersen, S.E., Newsome, W.T. \& Allman, J.M. (1981) Visual response properties of neurons in four extrastriate visual areas of the owl monkey (Aotus trivirgatus): a quantitative comparison of medial, dorsomedial, dorsolateral, and middle temporal areas. J. Neurophysiol., 45, 397-416.

Barton, J.S., Sharpe, J.A. \& Raymond, J.E. (1995) Retinotopic and directional defects in motion discrimination in humans with cerebral lesions. Ann. Neurol., 37, 665-675.

Beckers, G. \& Hömberg, V. (1992) Cerebral visual motion blindness: transitory akinetopsia induced by transcranial magnetic stimulation of human area V5. Proc. R. Soc. Lond. B Biol. Sci., 249, 173-178.

Beckers, G. \& Zeki, S. (1995) The consequences of inactivating areas V1 and V5 on visual motion perception. Brain, 118, 49-60.

Blanke, O., Landis, T., Safran, A.B. \& Seeck, M. (2002) Direction specific motion blindness induced by focal stimulation of human extrastriate cortex. Eur. J. Neurosci., 15, 2043-2048.

Blythe, I.M., Kennard, C. \& Ruddock, K.H. (1987) Residual vision in patients with retrogeniculate lesions of the visual pathways. Brain, 110, 887-905.

Brent, P.J., Kennard, C. \& Ruddock, K.H. (1994) Residual colour vision in a human hemianope: spectral responses and colour discrimination. Proc. $R$. Soc. Lond. B Biol. Sci., 256, 219-225.

Britten, K.H., Shadlen, M.N., Newsome, W.T. \& Movshon, J.A. (1992) The analysis of visual motion: a comparison of neuronal and psychophysical performance. J. Neurosci., 12, 4745-4765.
Celebrini, S. \& Newsome, W.T. (1995) Microstimulation of extrastriate area MST influences performance on a direction discrimination task. J. Neurophysiol., 73, 437-448.

Damasio, A.R., Tranel, D. \& Rizzo, M. (2000) Disorders of complex visual processing. In Mesulam, M.M. (ed.), Principles of Behavioral and Cognitive Neurology. Oxford University Press, New York, pp. 332-372.

Dubner, R. \& Zeki, S.M. (1971) Response properties and receptive fields of cells in an anatomically defined region of the superior temporal sulcus in the monkey. Brain. Res., 35, 528-532.

Dukelow, S.P., DeSouza, J.F., Culham, J.C., van den Berg, A.V., Menon, R.S. \& Vilis, T. (2001) Distinguishing subregions of the human MT+ complex using visual fields and pursuit eye movements. J. Neurophysiol., 86, 1991-2000.

Dumoulin, S.O., Bittar, R.G., Kabani, N.J., Baker, C.L., Le Goualher, G., Bruce Pike, G. \& Evans, A.C. (2000) A new anatomical landmark for reliable identification of human area V5/MT: a quantitative analysis of sulcal patterning. Cereb. Cortex, 10, 454-463.

Dupont, P., Orban, G.A., De Bruyn, B., Verbruggen, A. \& Mortelmans, L. (1994) Many areas in the human brain respond to visual motion. J. Neurophysiol., 72, 1420-1424.

Galetti, C., Fattori, P., Gamberini, M. \& Kutz, D.F. (1999b) The cortical visual area V6: brain location and visual topography. Eur. J. Neurosci., 11, 3922-3936.

Galetti, C., Fattori, P., Kutz, D.F. \& Gamberini, M. (1999a) Brain location and visual topography of cortical area V6A in the macaque monkey. Eur. $J$. Neurosci., 11, 575-582.

Gattass, R. \& Gross, C.G. (1981) Visual topography of the striate projection zone in the posterior superior temporal sulcus (MT) of the macaque. J. Neurophysiol., 46, 621-637.

Goebel, R., Khorram-Sefat, D., Muckli, L., Hacker, H. \& Singer, W. (1998) The constructive nature of vision: direct evidence from functional magnetic resonance imaging studies of apparent motion and motion imagery. Eur. J. Neurosci., 10, 1563-1573.

Goldstein, K. \& Gelb, A. (1918) Psychologische Analysen hirnpathologischer Fälle auf Grund von Untersuchungen Hirnverletzter. I. Abhandlung. Zur Psychologie des Optischen Wahrnehmungs- und Erkenungsvorganges. Z. die Gesamte Neurologie Psychiatrie, 41, 1-142.

Greenlee, M.W., Lang, H.J., Mergner, T. \& Seeger, W. (1995) Visual short term memory of stimulus velocity in patients with unilateral posterior brain damage. J. Neurosci., 15, 2287-2300.

Greenlee, M.W. \& Smith, A.T. (1997) Detection and discrimination of first and second order motion in patients with unilateral posterior brain damage. J. Neurosci., 17, 804-818.

Gross, B.L., Blake, R. \& Hirtis, E. (1998) Anisotropies in visual motion perception. A fresh look. J. Opt. Soc. Am. A Optics Images Sci. Vision, 15, 2003-2011.

Grüsser, O.J. \& Landis, T. (1991) Visual movement agnosia or motion blindnes: a rare clinical symptom. Visual Agnosias and Other Disturbances of Visual Perception and Cognition. Macmillan, Amsterdam, pp. 359-384.

Heeger, D.J., Boynton, G.M., Demb, J.B., Seidemann, E. \& Newsome, W.T. (1999) Motion opponency in visual cortex. J. Neurosci., 19, 7162-7174.

Hotson, J., Braun, D., Herzberg, W. \& Boman, D. (1994) Transcranial magnetic stimulation of extrastriate cortex degrades human motion direction discrimination. Vision Res., 34, 2115-2123.

Huk, A.C., Dougherty, R.F. \& Heeger, D.J. (2002) Retinotopy and functional subdivision of human areas MT and MST. J. Neurosci., 22, 7195-7205.

Huk, A.C., Ress, D. \& Heeger, D.J. (2001) Neuronal basis of the motion aftereffect reconsidered. Neuron, 32, 161-172.

de Jong, B.M., Shipp, S., Skidmore, B., Frackowiak, R.S. \& Zeki, S. (1994) The cerebral activity related to the visual perception of forward motion in depth. Brain, 117, 1039-1054.

Levinson, E. \& Sekuler, R. (1976) Adaptation alters perceived direction of motion. Vision Res., 16, 779-781.

Losey, F., Safran, A.B., Mermoud, C., Michel, C.M. \& Landis, T. (1998) Perception visuelle du mouvement. Etude normative. Klin. Monatsbl. Augenheilkd., 212, 379-381.

Malonek, D., Tootell, R.B. \& Grinvald, A. (1994) Optical imaging reveals the functional architecture of neurons processing shape and motion in owl monkey area MT. Proc. R. Soc. Lond. B Biol. Sci., 258, 109-119.

Mather, G. (1980) The movement aftereffect and a distribution-shift model for coding the direction of visual movement. Perception, 9, 379-392.

Maunsell, J.H. \& Newsome, W.T. (1987) Visual processing in monkey extrastriate cortex. Annu. Rev. Neurosci., 10, 363-401.

Maunsell, J.H. \& Van Essen, D.C. (1987) Topographic organization of the middle temporal visual area in the macaque monkey: representational biases and the relationship to callosal connections and myeloarchitectonic boundaries. J. Comp. Neurol., 266, 535-555. 
Nakayama, K. (1985) Biological image motion processing: a review. Vision Res., 25, 625-660.

Newsome, W.T. \& Paré, E.B. (1988) A selective impairment of motion perception following lesions to the middle temporal visual area (MT). J. Neurosci., 8, 2201-2211.

Newsome, W.T., Shadlen, M.N., Zohary, E., Britten, K.H. \& Movshon, J.A (1995) Visual motion: linking neuronal activity to psychophysical performance. In: Gazzaniga, M.E. (ed.), The cognitive neurosciences. MIT Press, Cambridge, pp. 401-414.

Parker, A.J. \& Newsome, W.T. (1998) Sense and the single neuron: probing the physiology of perception. Аппи. Rev. Neurosci., 21, 227-277.

Pasternak, T. \& Merigan, W.H. (1994) Motion perception following lesions of the superior temporal sulcus in the monkey. Cereb. Cortex, 4, $247-259$.

Plant, G.T., Laxer, K.D., Barbaro, N.M., Schiffman, J.S. \& Nakayama, K. (1993) Impaired visual motion processing in the contralateral hemifield following unilateral posterior cerebral lesions in humans. Brain, 116, 1303-1335.

Plant, G.T. \& Nakayama, K. (1993) The characteristics of residual motion perception in the hemifield contralateral to lateral occipital lesions in humans. Brain, 116, 1337-1353.

Poetzl, O. \& Redlich, E. (1911) Demonstration eines Falles von bilateraler Affektion beider Occipitallappen. Wiener Klinische Wochenschrift, 24, $517-518$.

Raymond, J.E. (1994) Directional anisotropy of motion sensitivity across the visual field. Vision Res., 34, 1029-1037.

Rudolph, K. \& Pasternak, T. (1999) Transient and permanent deficits in motion perception after lesions of cortical areas MT and MST in the macaque monkey. Cereb. Cortex, 9, 90-100.

Salzman, C.D., Britten, K.H. \& Newsome, W.T. (1990) Cortical microstimulation influences perceptual judgements of motion direction. Science, 346 174-177.

Salzman, C.D., Murasugi, C.M., Britten, K.H. \& Newsome, W.T. (1992) Microstimulation in visual area MT: effects on direction discrimination performance. J. Neurosci., 12, 2331-2355.

Schenk, T. \& Zihl, J. (1997) Deficits in global motion perception. Neuropsychologia, 35, 1289-1297.

Shadlen, M.N., Britten, K.H., Newsome, W.T. \& Movshon, J.A. (1996) A computational analysis of the relationship between neuronal and behavioral responses to visual motion. J. Neurosci., 16, 1486-1510.

Shipp, S., Blanton, M. \& Zeki, S. (1996) A visuo-somatomotor pathway through superior parietal cortex in the macaque monkey: cortical connections of areas V6 and V6A. Eur. J. Neurosci., 10, 3171-3193.
Shipp, S., de Jong, B.M., Zihl, J., Frackowiak, R.S. \& Zeki, S. (1994) The brain activity related to residual motion vision in a patient with bilateral lesions of V5. Brain, 117, 1023-1038.

Simoncelli, E.P. \& Heeger, D.J. (1998) A model of neuronal responses in visual area MT. Vision Res., 38, 743-761.

Spinelli, L., Blanke, O., Perrig, S., Lantz, G., Thut, G., Ducommun, C., Lazeyras, F., Gries, P., Slosman, D.O., Villemure, J.-G., Michel, C.M. \& Seeck, M. (2001) Comprehensive pre-surgical exploration for epilepsy using multimodal imagery and invasive recordings: a case report. Neuroimage, $\mathbf{1 3}$, S841.

Sunaert, S., Van Hecke, P., Marchal, G. \& Orban, G.A. (1999) Motionresponsive regions of the human brain. Exp. Brain Res., 127, 355-370.

Talairach, J. \& Tournoux, P. (1988) Co-Planar Stereotaxic Atlas of the Human Brain. Thieme, New York.

Tootell, R.B.H., Reppas, J.B., Kwong, K.K., Malach, R., Born, R.T., Brady, T.J., Rosen, B.R. \& Belliveau, J.W. (1995) Functional analysis of human MT and related visual cortical areas using magnetic resonance imaging. J. Neurosci., 15, 3215-3230.

Vaina, L.M. (1989) Selective impairment of visual motion interpretation following lesions of the right occipito-parietal area in humans. Biol. Cybern., 61, 347-359.

Vaina, L.M., Cowey, A., Eskew, R.T., LeMay, M. \& Kemper, T. (2001) Regional cerebral correlates of global motion perception: evidence from unilateral cerebral brain damage. Brain, 124, 310-321.

Van Essen, D.C., Maunsell, J.H. \& Bixby, J.L. (1981) The middle temporal visual area in the macaque: myeloarchitecture, connections, functional properties and topographic organization. J. Comp. Neurol., 199, 293-326.

Watson, J.D.G., Myers, R., Frackowiak, R.S., Hajnal, J.V., Woods, R.P., Mazziotta, J.C., Shipp, S. \& Zeki, S. (1993) Area V5 of the human brain: evidence from a combined study using positron emission tomography and magnetic resonance imaging. Cereb. Cortex, 3, 79-94.

Zeki, S.M. (1974) Functional organization of a visual area in the posterior bank of the superior temporal sulcus of the rhesus monkey. J. Physiol. (Lond.) 236, 549-573.

Zeki, S.M. (1991) Cerebral akinetopsia (visual motion blindness). Brain, 114, 811-824.

Zeki, S. \& ffytche, D.H. (1998) The Riddoch syndrome: insights into the neurobiology of conscious vision. Brain, 121, 25-45.

Zihl, J., von Cramon, D. \& Mai, N. (1983) Selective disturbance of movement vision after bilateral brain damage. Brain, 106, 313-340.

Zihl, J., von Cramon, D., Mai, N. \& Schmid, C. (1991) Disturbance of movement vision after bilateral posterior brain damage. Further evidence and follow up observations. Brain, 114, 2235-2252. 